LBNL-43259

UCB-PTH-99/21

JHU-TIPAC-99004

hep-th/9905122

May, 1999

\title{
Gaugino Masses in Modular Invariant Supergravity
}

\author{
Mary K. Gaillard, Brent Nelson \\ Department of Physics, University of California, and \\ Theoretical Physics Group, 50A-5101, Lawrence Berkeley National Laboratory, \\ Berkeley, CA 94720, USA \\ and \\ Yi-Yen $\mathrm{Wu}$ \\ Department of Physics and Astronomy, Johns Hopkins University, Baltimore, \\ Maryland 21218, USA
}

\begin{abstract}
We calculate gaugino masses in string-derived models with hidden-sector gaugino condensation. The linear multiplet formulation for the dilaton superfield is used to implement perturbative modular invariance. The contribution arising from quantum effects in the observable sector includes the term recently found in generic supergravity models. A much larger contribution is present if matter fields with Standard Model gauge couplings also couple to the Green-Schwarz counter term. We comment on the relation of our Kähler $U(1)$ superspace formalism to other calculations.
\end{abstract}

${ }^{*}$ This work was supported in part by the Director, Office of Energy Research, Office of High Energy and Nuclear Physics, Division of High Energy Physics of the U.S. Department of Energy under Contract DE-AC03-76SF00098 and in part by the National Science Foundation under grants PHY-95-14797 and PHY-94-04057. 


\section{Disclaimer}

This document was prepared as an account of work sponsored by the United States Government. While this document is believed to contain correct information, neither the United States Government nor any agency thereof, nor The Regents of the University of California, nor any of their employees, makes any warranty, express or implied, or assumes any legal liability or responsibility for the accuracy, completeness, or usefulness of any information, apparatus, product, or process disclosed, or represents that its use would not infringe privately owned rights. Reference herein to any specific commercial products process, or service by its trade name, trademark, manufacturer, or otherwise, does not necessarily constitute or imply its endorsement, recommendation, or favoring by the United States Government or any agency thereof, or The Regents of the University of California. The views and opinions of authors expressed herein do not necessarily state or reflect those of the United States Government or any agency thereof, or The Regents of the University of California.

Lawrence Berkeley Laboratory is an equal opportunity employer. 
It was recently pointed out [1], 2] that the super-Weyl anomaly of standard $N=1$ supergravity generates a gaugino mass proportional to the beta-function coefficient, which may solve the problem of small gaugino masses found in certain classes of models.

In this paper we consider a class of string-derived models [3]-[5] in which gaugino condensation occurs in a hidden sector with modular invariant couplings. That is, the field theoretic quantum anomaly that breaks invariance under the modular transformation (T-duality)

$$
T \rightarrow \frac{a T-i b}{i c T+d}, \quad a b-c d=1, \quad a, b, c, d \in Z
$$

is explicitly canceled by a universal Green-Schwarz (GS) counter term together with model-dependent string threshold corrections. Gaugino masses in these models were found in [5] to be suppressed with respect to the gravitino mass-although not as severely as in some gauge-mediated models [6]. However, in [5] a contribution was omitted that generates, among others, the term found in [1, 2n. In this paper we correct this omission. The additional correction is obtained by an analysis of the superspace expression for the loop correction, as well as by an explicit calculation using component fields and Pauli-Villars regularization. In these models the anomaly associated with the Kähler transformation (1) is explicitly canceled. Because Kähler and super-Weyl transformations are intimately connected in the Kähler $U(1)$ superspace formalism [7] that we use, one might expect the mass term found in [1, 2] to be absent in this class of models. However, this term has its origin in the running of the couplings from the string scale to the condensation scale, and is therefore independent of the string scale physics. In addition, we find a contribution that depends on the unknown couplings of matter fields in the GS term - a situation similar to the case for scalar masses discussed in [5].

In the linear supermultiplet formulation the dilaton $\ell$ is the lowest component of a vector superfield $L$ that satisfies the modified linearity condition

$$
-\left(\overline{\mathcal{D}}^{2}-8 R\right) L=W^{\alpha} W_{\alpha}, \quad-\left(\mathcal{D}^{2}-8 \bar{R}\right) L=\bar{W}_{\dot{\alpha}} \bar{W}^{\dot{\alpha}}, \quad L \mid=\ell,
$$


where the superfield $R$ is related to elements of the supervielbein, $W^{\alpha}$ is a Yang-Mills superfield strength, and the summation over gauge indices is suppressed. The Bianchi identity

$$
\left(\mathcal{D}^{2}-24 \bar{R}\right) W^{\alpha} W_{\alpha}-\left(\overline{\mathcal{D}}^{2}-24 R\right) \bar{W}_{\dot{\alpha}} \bar{W}^{\dot{\alpha}}=\text { total derivative }
$$

follows immediately from (22). To describe gaugino condensation [8, 9], a vector multiplet $V$ is introduced whose components include those of a linear multiplet $L$ as well as chiral and anti-chiral superfields $U, \bar{U}$ that are the (anti-)chiral projections of $V$ :

$$
-\left(\overline{\mathcal{D}}^{2}-8 R\right) V=U, \quad-\left(\mathcal{D}^{2}-8 \bar{R}\right) V=\bar{U},
$$

and are interpreted as condensate superfields for a strongly coupled (confined) hidden Yang-Mills sector: $U \simeq\left(W^{\alpha} W_{\alpha}\right)_{h}$. With this construction the superfield $U$ has the correct Kähler $U(1)$ weight as well as the correct constraint, that is, the counterpart of the Bianchi identity (3). This construction was generalized in [4] to the case of several gaugino condensates, and it was found that the results are dominated by the condensate of the gauge group $\mathcal{G}_{a}=\mathcal{G}_{+}$with the largest $\beta$-function coefficient $b_{a}=b_{+}$, where

$$
b_{a}=\frac{1}{8 \pi^{2}}\left(C_{a}-\frac{1}{3} C_{a}^{M}\right), \quad C_{a}^{M}=\sum_{A} C_{a}^{A},
$$

with $C_{a}$ and $C_{a}^{A}$ quadratic Casimir operators in the adjoint and matter representations, respectively. For this reason we include only a single condensate here. When both the condensate and the weakly coupled, unconfined Yang-Mills sectors are included, the linearity condition takes the form

$$
\begin{aligned}
& -\left(\overline{\mathcal{D}}^{2}-8 R\right) V=U+\sum_{a}\left(W^{\alpha} W_{\alpha}\right)_{a} \\
& -\left(\mathcal{D}^{2}-8 \bar{R}\right) V=\bar{U}+\sum_{a}\left(\bar{W}_{\dot{\alpha}} \bar{W}^{\dot{\alpha}}\right)_{a},
\end{aligned}
$$

We consider a class of orbifold compactifications with three untwisted moduli chiral superfields $T^{I}$ and matter chiral superfields $\Phi^{A}$. The Kälher potential is

$$
K=k(V)+\sum_{I} g^{I}+\sum_{A} e^{q_{I}^{A}}\left|\Phi^{A}\right|^{2}+O\left(\Phi^{4}\right), \quad g^{I}=-\ln \left(T^{I}+\bar{T}^{I}\right),
$$


where the parameters $q_{I}^{A}$ are the modular weights of $\Phi^{A}$, and the relevant part of the Lagrangian is

$$
\mathcal{L}_{\text {eff }}=\mathcal{L}_{1}+\sum_{a} \mathcal{L}_{a}, \quad \mathcal{L}_{1}=\mathcal{L}_{K E}+\mathcal{L}_{G S}+\mathcal{L}_{t h}+\mathcal{L}_{V Y}+\mathcal{L}_{\text {pot }}
$$

where

$$
\mathcal{L}_{K E}=\int \mathrm{d}^{4} \theta E[-2+f(V)], \quad k(V)=\ln V+g(V),
$$

contains the kinetic energy terms for the dilaton, chiral and gravity superfields, as well as the tree-level Yang-Mills terms. The functions $f(V), g(V)$ parameterize nonperturbative string effects. They satisfy the conditions

$$
V g^{\prime}(V)=f-V f^{\prime}(V), \quad g(0)=f(0)=0,
$$

which ensure that the Einstein term has canonical form [3], and that they vanish in weak coupling limit: $g^{2} / 2=\langle\ell\rangle=\langle V \mid\rangle \rightarrow 0$. The term

$$
\begin{aligned}
\mathcal{L}_{V Y} & =\frac{1}{8} \int \mathrm{d}^{4} \theta \frac{E}{R} U\left[b_{+}^{\prime} \ln \left(e^{-K / 2} U\right)+\sum_{\alpha} b^{\alpha} \ln \Pi^{\alpha}\right]+\text { h.c. }, \\
b_{a}^{\prime} & =\frac{1}{8 \pi^{2}}\left(C_{a}-C_{a}^{M}\right), \quad \sum_{\alpha} b^{\alpha}=\frac{1}{12 \pi^{2}} C_{+}^{M}
\end{aligned}
$$

is the generalization to supergravity [10, 11] of the Veneziano-Yankielowicz superpotential term [12], including [13] gauge invariant composite matter fields $\Pi^{\alpha}$, and

$$
\mathcal{L}_{\text {pot }}=\frac{1}{2} \int \mathrm{d}^{4} \theta \frac{E}{R} e^{K / 2} W\left(\Pi^{\alpha}, T^{I}\right)+\text { h.c. }
$$

is a superpotential for the matter condensates. In (11) $C_{+}^{M}$ refers to the confined matter superfields $\Phi^{A}$ of the strongly coupled sector.

The operators $\mathcal{L}_{a}$ are the quantum corrections from light field loops to the unconfined Yang-Mills couplings:

$$
\mathcal{L}_{a}=\frac{1}{64 \pi^{2}} \int d^{4} \theta \frac{E}{R}\left(W_{\alpha} P_{\chi}\left[f_{a}\left(\square_{\chi}\right)-B_{a}\right] W^{\alpha}\right)_{a}+\text { h.c. },
$$


where $\square_{\chi}^{-1}$ is the chiral superfield propagator [14]:

$$
\left\langle\square_{\chi}\right\rangle=\left\langle\square+\frac{1}{2} R \mathcal{D}^{2}+O(R \bar{R})\right\rangle,
$$

in our notation, $]^{\mathbb{N}}$ and $P_{\chi}$ is the chiral projection operator: $P_{\chi} W^{\alpha}=W^{\alpha}$, that reduces in the flat space limit to $(16 \square)^{-1} \overline{\mathcal{D}}^{2} \mathcal{D}^{2}$. The function [15]

$$
\begin{aligned}
B_{a} & =\sum_{I}\left(C-b_{a}^{I}\right) g^{I}+\left(C^{a}-C_{M}^{a}\right) k(V)+2 \sum_{A} C_{A}^{a} \ln \left(1+p_{A} V\right), \\
b_{a}^{I} & =C-C_{a}+\sum_{A}\left(1-2 q_{I}^{A}\right) C_{a}^{A}, \quad C=C_{E_{8}}=30
\end{aligned}
$$

determines the renormalized coupling constant [5, 10, 16] $g_{a}\left(\mu_{s}\right)$ at the string scale $\mu_{s}$ :

$$
\begin{aligned}
g_{a}^{-2}\left(\mu_{s}\right)= & \left\langle\frac{1+f}{2 \ell}-b_{a}^{\prime} k(\ell)+\sum_{A} \frac{C_{a}^{A}}{8 \pi^{2}} \ln \left(1+\ell p_{A}\right)\right. \\
& \left.-\sum_{I} \frac{b_{a}^{I}}{16 \pi^{2}} \ln \left[\left|\eta\left(i t^{I}\right)\right|^{2}\left(t^{I}+\bar{t}^{I}\right)\right]\right\rangle, \quad \mu_{s}=\left\langle e^{\frac{1}{2}(k-1)}\right\rangle,
\end{aligned}
$$

and the functions

$$
\frac{1}{8 \pi^{2}} f_{a}\left(\mu^{2}\right)=g_{a}^{2}\left(\mu^{2}\right)-g_{a}^{2}\left(\mu_{s}^{2}\right)
$$

govern the running of the gauge couplings from the string scale to the normalization scale $\mu^{2}=-<\square>$. $\mathcal{L}_{a}$ and $\mathcal{L}_{V Y}$ are anomalous under (11). This anomaly is canceled by two counter terms: the GS term [17]

$$
\begin{aligned}
\mathcal{L}_{G S} & =\int \mathrm{d}^{4} \theta E V V_{G S}, \quad b=\frac{C}{8 \pi^{2}}=b_{E_{8}}, \\
V_{G S} & =b \sum_{I} g^{I}+\sum_{A} p_{A} e^{\sum_{I} q_{I}^{A} g^{I}}\left|\Phi^{A}\right|^{2}+O\left(\Phi^{4}\right),
\end{aligned}
$$

\footnotetext{
${ }^{1}$ We set the background space-time curvature scalar $r$ to zero throughout this paper. A term proportional to $r \lambda \lambda$ would result in a contribution to the gaugino mass through a Weyl rescaling, but we find that such terms are suppressed by powers of $\mu^{-2}$ or $m^{-2}$ where $m$ is the Pauli-Villars mass introduced below.
} 
and the term induced by string loop corrections [18]

$$
\mathcal{L}_{t h}=-\frac{1}{64 \pi^{2}} \sum_{I} \int \mathrm{d}^{4} \theta \frac{E}{R} \ln \eta^{2}\left(i T^{I}\right)\left(b_{+}^{I} U+\sum_{a} b_{a}^{I}\left(W^{\alpha} W_{\alpha}\right)_{a}\right)+\text { h.c. }
$$

The parameters $b_{a}^{I}$ vanish for orbifold compactifications with no $N=2$ supersymmetry sector [19]. For $a=+$, the $q_{I}^{A}$ are modular weights of the confined matter superfields. Note that we have not introduced kinetic terms for the condensate superfield; that is, we are treating the condensate as static. A dynamical condensate has been studied [20] in the case of an $E_{8}$ gauge group, and it was found that the bound state masses are above the condensation scale; when these states are integrated out the theory reduces to the static case considered here.

To evaluate the gaugino masses, we set all matter fields to zero in the vacuum: $<\phi^{A}>=<\Phi^{A} \mid>=0$. First recall that "D-terms" like $\mathcal{L}_{G S}$ and $\mathcal{L}_{K E}$ can be cast in the form of "F-terms" by integration by parts:

$$
\mathcal{L}=\int \mathrm{d}^{4} \theta E \phi=-\frac{1}{16} \int \mathrm{d}^{4} \theta \frac{E}{R}\left(\overline{\mathcal{D}}^{2}-8 R\right) \phi+\text { h.c. }
$$

These and the remaining "F-terms" can be evaluated using the standard construction [7]

$$
\begin{aligned}
\mathcal{L} & =\frac{1}{2} \int \mathrm{d}^{4} \theta \frac{E}{R} \mathbf{r}=\sqrt{g}(s-r \bar{M})+\text { h.c. }+O\left(\psi_{\mu}\right), \\
r & =\mathbf{r}\left|, \quad s=-\frac{1}{4} \mathcal{D}^{2} \mathbf{r}\right|
\end{aligned}
$$

with

$$
\begin{aligned}
W^{\alpha} W_{\alpha} \mid & =-\lambda \lambda, \quad u=U|, \quad M=-6 R|=(\bar{M})^{*}, \quad \ell=V \mid, \\
t^{I} & =T^{I}\left|, \quad F^{I}=-\frac{1}{4} \mathcal{D}^{2} T^{I}\right|, \quad F_{u}=-\frac{1}{4} \mathcal{D}^{2} U \mid \\
-\frac{1}{4} \mathcal{D}^{2} W^{\alpha} W_{\alpha} \mid & =-\frac{1}{2}\left(F^{2}+i F \cdot \tilde{F}\right)-\bar{M} \lambda \lambda+2 \bar{\lambda} i \not D D \\
&
\end{aligned}
$$

For example,

$$
\mathcal{L}_{G S}=-\frac{1}{16} \int \mathrm{d}^{4} \theta \frac{E}{R}\left(\overline{\mathcal{D}}^{2}-8 R\right) V V_{G S}+\text { h.c. }
$$




$$
\begin{aligned}
& =\frac{1}{16} \int \mathrm{d}^{4} \theta \frac{E}{R}\left(\sum_{a}\left(W^{\alpha} W_{\alpha}\right)_{a} V_{G S}+V \overline{\mathcal{D}}^{2} V_{G S}\right)+\text { h.c. }+\cdots \\
& =\frac{b}{8} \sum_{I}\left(\bar{\lambda} i \not \supset \lambda g^{I}-\lambda \lambda F^{I} g^{I}\right)+\text { h.c. }+\cdots
\end{aligned}
$$

The evaluation of the component form of $\mathcal{L}_{1}$ is rather involved and has been carried

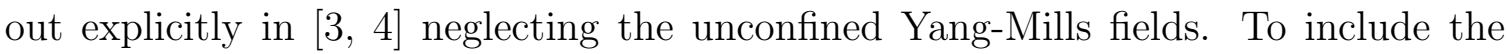
latter terms we need only make the substitutions

$$
u \rightarrow u-\sum_{a}(\lambda \lambda)_{a}, \quad F_{u} \rightarrow F_{u}-\frac{1}{4} \sum_{a} \mathcal{D}^{2}\left(W^{\alpha} W_{\alpha}\right)_{a}
$$

in those results. Using the vacuum values found in [4]:

$$
\left\langle F^{I}\right\rangle=0, \quad\langle\bar{M}\rangle=\frac{3}{4}\left\langle\left(b_{+}^{\prime} \bar{u}-4 \bar{W} e^{K / 2}\right)\right\rangle=\frac{3}{4} b_{+}\langle\bar{u}\rangle=-3 m_{\tilde{G}},
$$

the contribution of $\mathcal{L}_{1}$ to observable sector gaugino masses found in [5] is

$$
\mathcal{L}_{1} \ni \frac{1}{2 g_{s}^{2}} \bar{\lambda} i \not \supset \lambda+\frac{1}{16 \ell^{2}}\left(1+\ell g^{\prime}\right)\left(1+b_{+} \ell\right) \bar{u} \lambda \lambda+\text { h.c. },
$$

where $m_{\tilde{G}}$ is the gravitino mass, and

$$
g_{s}=\left\langle\sqrt{\frac{2 \ell}{1+f}}\right\rangle
$$

is the tree-level field theory coupling constant. The requirement that the vacuum energy vanishes gives the condition [4]

$$
\left\langle\left(1+\ell g^{\prime}\right)\left(1+b_{+} \ell\right)^{2}\right\rangle=3\left\langle\ell^{2}\right\rangle b_{+}^{2},
$$

so that, taking into account gauge coupling renormalization, one gets a contribution to the gaugino mass [5]

$$
m_{a}^{(1)}\left(\mu_{c}\right)=-\left\langle\frac{3 g_{a}^{2}\left(\mu_{c}\right) b_{+}^{2} \bar{u}}{8\left(1+b_{+} \ell\right)}\right\rangle=\frac{3 g_{a}^{2}\left(\mu_{c}\right) b_{+}}{2\left(1+b_{+} \ell\right)} m_{\tilde{G}},
$$

where $\mu_{c}=|u|^{\frac{1}{3}}$ is the condensation scale in reduced Planck units. A gravitino mass in the $\mathrm{TeV}$ range requires $b_{+} \simeq 1 / 30$, so this contribution to the gaugino masses 
is quite small, although it is possible that two-loop renormalization effects between the condensation scale and the weak scale can bring masses of this order within experimental bounds 21.

To evaluate (13) we drop [2] terms of order $\left\langle R \bar{R} / \square>=-m_{\tilde{G}}^{2} / 4 \mu^{2}\right.$ to obtain

$$
\left\langle\left[\mathcal{D}^{2}, f\left(\square_{\chi}\right)\right]\right\rangle \Phi=\left\langle 8 \bar{R}\left[f^{\prime}\left(\square_{\chi}\right) \square+R\left\{f^{\prime}\left(\square_{\chi}\right)+f^{\prime \prime}\left(\square_{\chi}\right) \square\right\} \mathcal{D}^{2}\right]\right\rangle \Phi .
$$

where $\Phi$ is chiral; only the first term on the right hand side contributes to gaugino masses; this is the contribution found in [1, 2]. To lowest order in perturbation theory,

$$
\begin{aligned}
& f_{a}(\square)=\left(3 C_{a}-C_{a}^{M}\right) \ln \left(\square / \mu_{s}^{2}\right), \\
& \left\langle\left[\mathcal{D}^{2}, f_{a}(\square)\right]\right\rangle \lambda_{a}=8\langle\bar{R}\rangle\left(3 C_{a}-C_{a}^{M}\right) \lambda_{a} .
\end{aligned}
$$

In addition we have:

$$
\left\langle\mathcal{D}^{2} B_{a} \mid\right\rangle=-\left\langle 4 \sum_{I} F^{I} \frac{\partial}{\partial T^{I}} B_{a}\left|+(\bar{U}-8 V \bar{R}) \frac{\partial}{\partial V} B_{a}\right|\right\rangle .
$$

Using the vacuum values (25), we obtain

$$
\begin{aligned}
-\frac{1}{2} m_{a}^{(2)}\left(\mathcal{L}_{a}\right)= & \frac{1}{128 \pi^{2}}\left\langle\mathcal{D}^{2}\left[f_{a}\left(\square_{\chi}\right)-B_{a}\right]\right\rangle \\
= & -\frac{\bar{u}}{128 \pi^{2}}\left\{\left(3 C_{a}-C_{M}\right) b_{+}-\left(1+b_{+} \ell\right) \times\right. \\
& {\left.\left[\ell^{-1}\left(\ell g^{\prime}+1\right)\left(C_{a}-C_{M}\right)+2 \sum_{A} C_{a}^{A} \frac{p_{A}}{\left(1+\ell p_{A}\right)}\right]\right\}, }
\end{aligned}
$$

giving for the full contribution at the condensation scale $\mu_{c}$

$$
\begin{gathered}
\mathcal{L}_{1}+\sum_{a} \mathcal{L}_{a} \ni \sum_{a}\left[\frac{1}{2 g_{a}^{2}\left(\mu_{c}\right)}(\bar{\lambda} i \not \supset \lambda)_{a}+\frac{M_{a}}{16} \bar{u}(\lambda \lambda)_{a}+\text { h.c. }\right], \\
M_{a}=\left(1+b_{+} \ell\right)\left[\ell^{-2}\left(1+\ell g^{\prime}\right)\left(1+b_{a}^{\prime} \ell\right)+\sum_{A} \frac{C_{a}^{A} p_{A}}{4 \pi^{2}\left(1+\ell p_{A}\right)}\right]-3 b_{a} b_{+} \cdot
\end{gathered}
$$

We now obtain for the gaugino masses

$$
m_{a}\left(\mu_{c}\right)=\frac{g_{a}^{2}\left(\mu_{c}\right)}{2}\left[\frac{3 b_{+}\left(1+b_{a}^{\prime} \ell\right)}{1+b_{+} \ell}-3 b_{a}+\sum_{A} \frac{C_{a}^{A} p_{A}\left(1+b_{+} \ell\right)}{4 \pi^{2} b_{+}\left(1+\ell p_{A}\right)}\right] m_{\tilde{G}} .
$$


Next we explicitly calculate the gaugino masses using a Pauli-Villars (PV) regularization that has been formulated [22] for supergravity Lagrangians with the dilaton in a chiral multiplet. For present purposes, we need only consider the regulation of loops containing gauge-charged fields. Because the results below depend only on the Kähler potential for the PV fields and their couplings to the GS term, it is straightforward to transcribe the analysis to the case where the dilaton is described by a linear supermultiplet.

To regulate loop corrections to the Yang-Mills self-energy, one needs gauge-charged PV chiral supermultiplets: $Z^{A}$ with signature $\eta_{A}$, that transform under gauge transformations according to representations $R_{A} ; Y_{A}$ with the same signature that transform according to the conjugate representation $\bar{R}_{A}$; and $\Phi_{\alpha}^{a}$, with signature $\eta_{\alpha}^{a}$, that transforms according to the adjoint representation of the gauge group. In order to cancel the logarithmic divergences in the Yang-Mills self-energy, the Casimirs $\operatorname{Tr}_{R_{A}}\left(T^{a} T^{b}\right)=\delta_{a b} C_{R_{A}}^{a}$, and the signatures of the PV fields must satisfy

$$
\sum_{A} \eta_{A} C_{R_{A}}^{a}=-C_{a}^{M}, \quad \sum_{\alpha} \eta_{\alpha}^{a}=3
$$

The Kähler potential for these fields takes the form (setting light gauge-charged fields to zero in the background)

$$
\begin{aligned}
K_{P V}= & \sum_{A}\left[g_{A}^{Z}(V) e^{\sum_{I} \alpha_{A}^{I} g^{I}}\left|Z^{A}\right|^{2}+g_{A}^{Y}(V) e^{\sum_{I} \beta_{A}^{I} g^{I}}\left|Y_{A}\right|^{2}\right] \\
& +\sum_{a, \alpha} g_{\alpha}(V) e^{\alpha_{\alpha} \sum_{I} g^{I}}\left|\Phi_{\alpha}^{a}\right|^{2} \equiv \sum_{n} g_{n}(V) G_{n}(T)\left|\Phi^{n}\right|^{2},
\end{aligned}
$$

The $V$-dependence of $K_{P V}$ requires an additional term $\mathcal{L}_{P V}=\int d^{4} \theta E f_{P V}(V)$ in the Lagrangian, where $f_{P V}$ is related to $K_{P V}$ by the differential equation in (10) that relates $f$ to $g$ :

$$
f_{P V}(V)=\sum_{n} f_{n}(V) G_{n}(T)\left|\Phi^{n}\right|^{2}, \quad V g_{n}^{\prime}=f_{n}-V f_{n}^{\prime}, \quad \Phi^{n}=Z^{A}, Y_{A}, \Phi_{\alpha}^{a} .
$$

The component Lagrangian can be obtained following the methods outlined in [3]. As shown in [15], supersymmetry of the modular anomaly from field theory quantum 
corrections imposes constraints on the parameters in (37):

$$
\begin{aligned}
1 & =\sum_{\alpha} \eta_{\alpha}^{a} \alpha_{\alpha}, \quad \sum_{A} \eta_{A}\left(\alpha_{A}^{I}+\beta_{A}^{I}\right) C_{R_{A}}^{a}=-\sum_{A} C_{a}^{A} q_{I}^{A}, \\
0 & =\sum_{A} \eta_{A}\left(\ln h_{A}^{Z}+\ln h_{A}^{Y}\right) C_{R_{A}}^{a}, \quad \sum_{\alpha} \eta_{\alpha}^{a} \ln h_{\alpha}=k, \\
h_{n}(V) & =f_{n}(V)+g_{n}(V) .
\end{aligned}
$$

in agreement with the requirements for full one-loop regularization [22]. Among the fields $Z^{A}$ there is a subset $\tilde{Z}_{\alpha}^{A}$ with the same modular weights and the same gauge couplings as the light fields $\Phi^{A}$. If the parameters $p_{A}$ in the Green-Schwarz term are nonvanishing, we also require

$$
\begin{aligned}
V_{G S}^{P V} & =\sum_{A, \alpha} p_{A} e^{\sum_{I} q_{I}^{A} g^{I}}\left|\tilde{Z}_{\alpha}^{A}\right|^{2}, \quad g_{A}^{\tilde{Z}}=h_{A}^{\tilde{Z}}=1, \\
\alpha_{A}^{\tilde{Z}^{I}} & =q_{I}^{A}, \quad \sum_{\alpha} \eta_{\alpha}^{\tilde{Z}^{A}}=-1 .
\end{aligned}
$$

The potential for the PV scalar fields $\phi^{n}=\phi^{a}, z^{A}, y_{A}$ is

$$
\begin{aligned}
V= & \frac{1}{16 \ell^{2}}\left(\ell g^{\prime}(\ell)+1\right)\left|u\left(1+b_{+} \ell\right)-4 \ell W^{P V} e^{K / 2}\right|^{2} \\
& -\frac{3}{16}\left|b_{+} u-4 W^{P V} e^{K / 2}\right|^{2}+\hat{K}_{n \bar{m}} F^{n} \bar{F}^{\bar{m}}
\end{aligned}
$$

where

$$
\begin{aligned}
-\bar{F}^{\bar{m}} & =\hat{K}^{n \bar{m}}\left\{\frac{u}{4} G_{n} \bar{\phi}^{n}\left[\left(p_{n}-b_{+}\right) h_{n}+h_{n}^{\prime}\left(1+\ell b_{+}\right)\right]+e^{K / 2} W_{n}^{P V}\right\}+O\left(\phi_{m}^{2}\right), \\
\hat{K}_{n \bar{m}} & =\left(\hat{K}^{n \bar{m}}\right)^{-1}=G_{n} h_{n}\left[1+p_{n} \ell\right] \delta_{n \bar{m}}, \quad p_{n \neq \tilde{Z}}=0 .
\end{aligned}
$$

The superpotential $W^{P V}$ contains quadratic terms in the PV fields that give their masses. These give rise to "B-terms" in the potential (41) which take the form

$$
\begin{aligned}
V_{B} & =\frac{1}{2} \sum_{m, n} \mu_{m n} a_{m} \phi^{m} \phi^{n}+\text { h.c., } \quad \mu_{m n}=e^{K / 2} W_{m n}^{P V}, \\
a_{n} & =e^{K / 2} \frac{\bar{u}}{4}\left\{b_{+} \ell+\left(1+b_{+} \ell\right)\left[\frac{2 p_{n}}{1+p_{n} \ell}+2 \frac{h_{n}^{\prime}}{h_{n}}-\frac{1}{\ell}\left(\ell g^{\prime}+1\right)\right]\right\} .
\end{aligned}
$$


The first term in this expression is independent of the PV Kähler potential - i.e., of the effective cut-offs - and of the details of the supersymmetry breaking mechanism. It is precisely the contribution found in [1, 2]. Here it arises from the presence of the Kähler potential in the condensate Lagrangian (11) as dictated by local supersymmetry. As noted previously [23], the structure of this term embeds the evolution of the gauge coupling constant from the string scale to the condensation scale. The PV Lagrangian also contains the terms (in four-component spinor notation)

$$
\begin{aligned}
\mathcal{L}_{P V} \ni & \sum_{n} \hat{K}_{n \bar{n}}\left[-\partial_{a} \bar{\phi}^{\bar{n}} \partial^{a} \phi^{n}+i \bar{\chi}_{L}^{\bar{n}} \not D \chi_{L}^{n}+\sqrt{2} i\left(\bar{\lambda}_{R}^{a}\left(\bar{\phi}^{\bar{n}} T_{a} \chi_{L}^{n}\right)+\text { h.c. }\right)\right] \\
& -\sum_{n, m}\left[\mu_{m n}^{2}\left|\phi^{n}\right|^{2}+\frac{1}{2} \mu_{m n}\left(\bar{\chi}_{R}^{n} \chi_{L}^{m}+\text { h.c. }\right)\right]
\end{aligned}
$$

In terms of the normalized fields $\Phi_{r}^{n}=\left(\hat{K}^{\frac{1}{2}}\right)_{n \bar{n}} \Phi^{n}$, the interaction terms are

$$
\begin{aligned}
\mathcal{L}_{Y u k} & =\sqrt{2} i \sum_{n} \bar{\lambda}_{R}^{a}\left(\bar{\phi}_{r}^{\bar{n}} T_{a} \chi_{r L}^{n}\right)+\text { h.c., } \quad V=\frac{1}{2} \sum_{m, n} m_{m} a_{m} \phi_{r}^{m} \phi_{r}^{n}+\text { h.c. }, \\
m_{n}^{2} & =\hat{K}^{n \bar{n}} \hat{K}^{m \bar{m}} \mu_{m n}^{2},
\end{aligned}
$$

where $m_{n}$ is the mass of $\Phi_{r}^{n}$. The Feynman amplitude $F_{a}=-i<\lambda_{R}^{a}\left|\mathcal{L}_{\text {eff }}\right| \lambda_{L}^{a}>$ for $\lambda_{L} \rightarrow \chi_{L}^{n}+\bar{\phi}^{\bar{n}} \rightarrow \phi^{m}+\bar{\chi}_{R}^{\bar{m}} \rightarrow \lambda_{R}$ gives a contribution

$$
\begin{aligned}
\mathcal{L}_{\text {eff }} & \ni-\frac{1}{2} m_{a} \bar{\lambda}_{R}^{a} \lambda_{L}^{a}+\text { h.c. }=\frac{i}{2} F_{a} \bar{\lambda}_{R}^{a} \lambda_{L}^{a}+\text { h.c. } \\
-\frac{1}{2} m_{a}^{(2)} & =-i \sum_{n} \eta_{n} C_{n}^{a} \int \frac{d^{4} p}{(2 \pi)^{4}} \frac{a_{n} m_{n}^{2}}{\left(p^{2}-m_{n}\right)^{3}}=-\sum_{n} \eta_{n} \frac{C_{n}^{a} a_{n}}{32 \pi^{2}},
\end{aligned}
$$

Using the constraints (36), (39) and (40), this reduces to the result found in (33).

Aside from the renormalization of the coupling constants, there are three contributions from $\mathcal{L}_{a}$ in (35). The first, proportional to $b_{a}^{\prime}$, gives a negligible correction to (29). The second term modifies the result (29) of [5] by a factor (neglecting $b_{+} \ell \sim .03$ )

$$
\eta_{a} \simeq\left(1-b_{a} / b_{+}\right) \simeq[0.6,1.1,1.8] \quad \text { for } \quad \mathcal{G}_{a}=[S U(3), S U(2), U(1)]
$$

if we assume just the MSSM contribution to the $\beta$-functions. The third term depends on the unknown parameters $p_{A}$. It was found in [5] that the squark, slepton and Higgs 
masses $m_{s}$ also depend on these parameters. If the matter fields decouple from the GS term, one has

$$
p_{A}=0, \quad m_{s}=m_{\tilde{G}},
$$

and the full correction to the gaugino masses is given by (47). If the GS term is proportional to Kähler potential we get

$$
p_{A}=b, \quad m_{s} \approx 10 m_{\tilde{G}}
$$

Analyses of dynamical symmetry breaking in the MSSM favor smaller masses for at least the stop and Higgs particles. Another possibility is that the GS term depends only on the metric of the compact 6-manifold, in which case it couples only to untwisted fields:

$$
p_{A}^{u n t w}=b, \quad m_{s}^{u n t w} \approx 10 m_{\tilde{G}}, \quad p_{A}^{t w}=0, \quad m_{s}^{t w}=0,
$$

resulting in a mass hierarchy among generations as has been proposed by some authors [24]. For a single Standard Model generation, $\sum_{A} C_{a}^{A}=2$, so in this scenario, with $n$ untwisted generations, the last term in (35) dominates, and one gets the following gaugino masses at the condensation scale:

$$
m_{a}\left(\mu_{c}\right) \approx-\frac{n \alpha_{a}\left(\mu_{c}\right) b\left(1+\ell b_{+}\right)}{\pi b_{+}(1+\ell b)} m_{\tilde{G}} \approx-\frac{10 n \alpha_{a}\left(\mu_{c}\right)}{\pi} m_{\tilde{G}}
$$

Finally, we address the generality of the result (34) in the broader context of the class of string-derived models that we are considering. It would be modified if modular invariance is broken by string nonperturbative effects, such as a modulidependence of the functions $g(V), f(V)$ as was found for a particular orbifold [25]. Modular invariance of the effective Lagrangian for the condensate ensures that the moduli are stabilized at one of the two self-dual points in the fundamental domain: $t^{I}=1, e^{i \pi / 6}$. Together with the condition that the vacuum energy vanishes, this assures that their F-components vanish in the vacuum: $\left\langle F^{I}\right\rangle=0$. The potential for the moduli and the dilaton [4] is

$$
16 \ell^{2} V=\left(1+\ell \frac{d g}{d \ell}\right)\left|\left(1+b_{+} \ell\right) u_{+}\right|^{2}
$$




$$
-3 \ell^{2}\left|b_{+} u_{+}\right|^{2}+4 \ell^{2}(1+b \ell) \sum_{I}\left|\frac{F^{I}}{\operatorname{Re} t^{I}}\right|^{2},
$$

so the condition (28), responsible for the suppression of gaugino masses if $p_{A}=0$, holds only to corrections of order $\left(<F^{I}>\right)^{2}$. If $<F^{I}>\neq 0$, there are additional corrections to gaugino masses from:

$$
\mathcal{L}_{t h}+\mathcal{L}_{G S}+\sum_{a} \mathcal{L}_{a} \ni-\sum_{a, I} \frac{b_{a}^{I}}{32 \pi^{2}} F^{I}\left[\frac{\eta^{\prime}\left(t^{I}\right)}{\eta\left(t^{I}\right)}-\frac{1}{4 \operatorname{Re} t^{I}}\right] .
$$

Since the term in brackets vanishes at the self-dual points, both corrections are of order $\left.\left(<F^{I}\right\rangle\right)^{2}$, and will be small if the moduli are stabilized near the self-dual points. Moreover, the second contribution is absent in the $Z_{3}, Z_{7}$ orbifolds that appear promising for model building [26]. The condition (28) provides several phenomenologically desirable features [5] of our model, namely moduli masses much larger than the gravitino mass:

$$
m_{\ell} \approx 50 m_{t^{I}} \approx 10^{3} m_{\tilde{G}}
$$

and a suppression of the axion decay constant by a factor of about 50 with respect to earlier estimates [27]. Moreover, the result that $\left\langle F^{I}\right\rangle=0$ avoids a potential source of unwanted flavor-changing neutral currents. A detailed analysis of the phenomenology of this class of models will be given elsewhere [21].

In concluding, we wish to emphasize that the gaugino mass contribution equal to $m_{0}=\beta\left(g^{2}\right) m_{\tilde{G}} / 2 g^{2}$ is a model independent result in our Kähler $U(1)$ superspace formalism, in agreement with the assertion made in [2]. In the formalism of [1], the auxiliary field of the supergravity multiplet differs from the field $M$ used here by a Weyl rotation that depends on the Kähler potential. As a result, the analogous term that they find is not model independent; for example they get no contribution in no-scale models [28]. In our formalism, $m_{0}$ in this case is exactly canceled by the contribution from $B_{a}$ in (13). Consider for example the simplest no-scale model with Kähler potential

$$
K=-3 \ln \left(T+\bar{T}-\sum_{A}\left|\Phi^{A}\right|^{2}\right), \quad<\phi^{A}>=0, \quad<W>\neq 0
$$


e.g. $W=\Phi^{3}+$ constant. In this model (with no cancellation of the modular anomaly)

$$
B_{a}=\left(C_{a}-\frac{1}{3} C_{a}^{M}\right) K, \quad\left\langle\mathcal{D}^{2} B_{a} \mid\right\rangle=-\left\langle 4 F^{T} \frac{\partial}{\partial T} B_{a}\right\rangle \mid,
$$

the vacuum values satisfy

$$
F^{T} K_{T}=-3 e^{K / 2} W, \quad R \mid=\frac{1}{2} e^{K / 2} W,
$$

and we obtain

$$
\left\langle\mathcal{D}^{2}\left[f_{a}\left(\square_{\chi}\right)-B_{a}\right]\right\rangle=0,
$$

in agreement with [28]. We find the same cancellation in a PV calculation for this model.

\section{Acknowledgements}

We thank Lisa Randall and Tom Taylor for discussions. This work was supported in part by the Director, Office of Energy Research, Office of High Energy and Nuclear Physics, Division of High Energy Physics of the U.S. Department of Energy under Contract DE-AC03-76SF00098 and in part by the National Science Foundation under grants PHY-95-14797 and PHY-94-04057.

\section{References}

[1] L. Randall and R. Sundrum MIT-CTP-2788, hep-th/9810155.

[2] G. Giudice, M. Luty, H. Murayama and R. Rattazzi, JHEP 9812, 027 (1998).

[3] P. Binétruy, M. K. Gaillard and Y.-Y. Wu, Nucl. Phys. B481 (1996) 109.

[4] P. Binétruy, M. K. Gaillard and Y.-Y. Wu, Nucl. Phys. B493 (1997) 27.

[5] P. Binétruy, M. K. Gaillard and Y.-Y. Wu, Phys. Lett. B412 (1997) 288. 
[6] M. Dine and D. MacIntire, Phys. Rev. D46 (1992) 2594;

T. Banks, D. Kaplan and A. Nelson, Phys. Rev. D49 (1994) 779.

[7] P. Binétruy, G. Girardi, R. Grimm and M. Müller, Phys. Lett. B189 (1987) 389;

P. Binétruy, G. Girardi and R. Grimm, preprint LAPP-TH-275/90.

[8] C.P. Burgess, J.-P. Derendinger, F. Quevedo and M. Quirós, Phys. Lett. B348 (1995) 428.

[9] P. Binétruy, M.K. Gaillard and T.R. Taylor, Nucl. Phys. B455 (1995) 97.

[10] T.R. Taylor, Phys. Lett. B164 (1985) 43.

[11] P. Binétruy and M.K. Gaillard, Phys. Lett. B232 (1989) 82.

[12] G. Veneziano and S. Yankielowicz, Phys. Lett. B113 (1982) 231.

[13] T.R. Taylor, G. Veneziano and S. Yankielowicz, Nucl. Phys. B218 (1983) 493; D. Lüst and T.R. Taylor. Phys. Lett. B253 (1991) 335.

[14] S.J. Gates, M. Grisaru, M. Roček and W. Siegel, Superspace (Benjamin/Cummings, 1983).

[15] M.K. Gaillard and T.R. Taylor, Nucl. Phys. B381 (1992) 577.

[16] V.S. Kaplunovsky and J. Louis, Nucl. Phys. B444 (1995) 191.

[17] G.L. Cardoso and B.A. Ovrut, Nucl. Phys. B369 (1993) 315;

J.-P. Derendinger, S. Ferrara, C. Kounnas and F. Zwirner, Nucl. Phys. B372 (1992) 145.

[18] L.J. Dixon, V.S. Kaplunovsky and J. Louis, Nucl. Phys. B355 (1991) 649.

[19] I. Antoniadis, K.S. Narain and T.R. Taylor, Phys. Lett. B267 (1991) 37.

[20] Y.-Y. Wu, Berkeley preprint LBNL-39441, UCB-96/42, hep-th/9610089, and paper in preparation. 
[21] B. Nelson, in preparation.

[22] M.K. Gaillard, Phys.Lett. B342: 125 (1995), Phys. Rev. D58: 105027 (1998) and paper in preparation.

[23] P. Binétruy and M.K. Gaillard, Nucl. Phys. B358, 121 (1991).

[24] A.G. Cohen, D.B. Kaplan and A.E. Nelson, Phys. Lett. B388 (1996) 588;

A. Pomarol and D. Tommasini, Nucl. Phys. B466 (1996) 3;

G. Dvali and A. Pomarol, Phys. Rev. Lett. 77 (1996) 3728.

[25] E. Silverstein, Phys. Lett. B396 (1997) 91.

[26] L.E. Ibáñez, H.-P. Nilles and F. Quevedo, Phys. Lett. B187, 25 (1987), A. Font, L. Ibáñez, F. Quevedo and A. Sierra, Nucl. Phys. B331, 421 (1990).

[27] K. Choi and J.E. Kim, Phys. Lett. B154 (1985) 393 and B165 (1985) 71; T. Banks and M. Dine, Phys. Rev. D 50 (1994) 7454.

[28] L. Randall and R. Sundrum, private communication. 